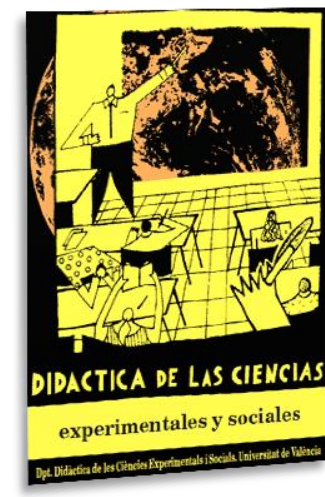

\title{
Educación para una ciudadanía comprometida en la enseñanza de las Ciencias Sociales: ¿Qué piensa el alumnado de un profesor innovador sobre su aprendizaje?
}

\author{
Education for a committed citizenship in the teaching \\ of Social Sciences: What do the students think in \\ relation to their learning with an innovative teacher?
}

DOI: $10.7203 / D C E S .35 .12572$

\author{
Laura Lucas Palacios \\ Universidad de Palencia, laura.lucas.palacios@uva.es \\ Emilio José Delgado Algarra \\ Universidad de Huelva, emilio.delgado@ddcc.uhu.es
}

\begin{abstract}
RESUMEN: En el presente artículo se presentan los resultados de una investigación que se enmarca en la tesis doctoral "la enseñanza de educación para la ciudadanía a partir de la educación patrimonial en las clases de Ciencias Sociales: un estudio de caso"; centrándonos en los bloques del sistema de categorías vinculados con «qué se enseña» sobre educación para la ciudadanía en las clases de Ciencias Sociales en el contexto de la Educación Secundaria. Para ello, con el apoyo del programa de análisis cualitativo ATLAS.ti, se lleva a cabo una categorización de las informaciones transcritas de la entrevista de concepciones del docente, del registro de audio sobre el desarrollo de las clases, de los grupos de discusión discente y de la entrevista final de reflexión docente. El caso de estudio muestra un docente comprometido con las problemáticas sociales actuales, que busca que su alumnado pueda desarrollar un pensamiento crítico que le lleve a comprometerse y participar en su realidad.
\end{abstract}

Palabras Clave: didáctica de las ciencias sociales, educación para la ciudadanía, educación patrimonial, innovación educativa, análisis cualitativo.

ABSTRACT: This paper presents the results of a research that is part of the doctoral thesis "teaching education for citizenship from heritage education in social science classes: a case study"; focusing on the sections of the system of categories linked to "what is taught" about citizenship education in Social Sciences classes in the context of Secondary Education. For this, along with the support of the qualitative analysis program ATLAS.ti, a categorization of the transcribed information of the teacher's conception interview, of the audio record on the development of the classes, of the student's discussion groups and of the final teacher reflection interview is carried out. The case study shows a teacher committed to current social problems, which seeks that their students can develop a critical thinking that leads them to engage and participate in their reality.

KEYWORDS: didactic of social sciences, citizenship education, heritage education, educational innovation, qualitative analysis.

Fecha de recepción: junio de 2018

Fecha de aceptación: noviembre de 2018

Este artículo forma parte del proyecto de investigación EPITEC "Educación Patrimonial para la inteligencia territorial y emocional de la ciudadanía” (MINECO/FEDER, con código de referencia EDU2015-67953-P). 


\section{INTRODUCCIÓN Y JUSTIFICACIÓN DEL TEMA DE ESTUDIO}

"La educación tiene que adaptarse en todo momento a los cambios de la sociedad, sin por ello dejar de transmitir el saber adquirido, los principios y los frutos de la experiencia" (Delors, 1996).

Habitualmente se plantea que la escuela y el sistema educativo en su conjunto deben tener un papel activo en la sociedad, aunque se podría reflexionar sobre si ese papel debe servir para transformar el funcionamiento general de la sociedad o para adaptar y permitir a los futuros ciudadanos y ciudadanas su participación activa en ella. Los cambios sociales, económicos, culturales, políticos..., a los que se está viendo sometido el mundo que conocemos, están dando lugar a que la comunidad educativa y la comunidad local estrechen lazos para dar respuestas acordes a las necesidades que se demandan hoy, surgiendo así nuevos planteamientos educativos.

La investigación de referencia se estructura en torno a tres grandes categorías: enseñanza del patrimonio, enseñanza de la ciudadanía y enseñanza de la educación para la ciudadanía a través del patrimonio. En relación con el presente artículo, dentro de la segunda categoría y tomando como fuentes principales Eurydice (2012) y la Evaluation of Educational Achievement [IEA] (Schulz et al., 2008), nos centraremos en la subcategoría "Qué se enseña de educación para la ciudadanía en CCSS" que se encontraría estructurado en torno a los siguientes indicadores: derechos individuales y conocimiento de las instituciones estatales y civiles, principios cívicos, identidades cívicas y participación cívica. En este sentido, Derechos individuales y conocimientos de las instituciones estatales y civiles guardan una relación con el conocimiento en torno a los mecanismos que tiene el estado para sustentar los contratos cívicos (relaciones entre ciudadanos/as), centrándose en las instituciones estatales para cuestiones relacionadas con la representación cívica y la legislación, y las instituciones civiles para la mediación entre instituciones estatales y los ciudadanos/as. Los principios cívicos guardan relación con los fundamentos éticos que comparte una sociedad (igualdad, libertad, cohesión social, dignidad y justicia). Las identidades cívicas, entendidas como múltiples y articuladas, incluyen las funciones del individuo en interacción (interconexiones cívicas) y las percepciones de éste sobre dichas funciones (autoimagen cívica). La participación cívica, por otra parte, hace referencia a las acciones llevadas a cabo por los individuos en su comunidad y se centra en cuestiones relacionadas con la toma de decisiones y la influencia en la comunidad.

Definidos estos conceptos, resulta necesario destacar que la educación para la ciudadanía se ha convertido en una de las principales finalidades de la enseñanza de las Ciencias Sociales, algo que se hace especialmente patente bajo el prisma de una didáctica crítica. Por lo demás, como indican Delgado-Algarra y Estepa-Giménez (2017), en el campo de la investigación didáctica, cada vez es mayor el interés por conocer el pensamiento y la práctica del profesorado de Ciencias Sociales en relación con la educación para una ciudadanía activa, participativa y democrática.

Por otra parte, algunas investigaciones, a las que alude Tutiaux-Guillon (2009), han demostrado que, frecuentemente, los deseos del profesorado de formar alumnos/as en actitudes democráticas son divergentes con su propia práctica. Igualmente, De Alba (2009) afirma que el profesorado cuestiona la capacidad de su alumnado para trabajar este tipo de contenidos y presenta cierta inseguridad a la hora de enseñarlos, debido a que no cuenta con una preparación específica; suponiéndole incluso un cuestionamiento de su propio saber y de su identidad profesional como docente, ya que se requiere especialmente que asuma su función como educador, constituyéndose como un modelo de adulto de referencia para el alumnado.

Por ello, en esta publicación se llevará a cabo un análisis sobre educación para la ciudadanía a partir de la revisión/observación de las clases de un profesor de secundaria. Se trata de un estudio realizado en el marco del desarrollo de la tesis doctoral La enseñanza de Educación para la Ciudadanía a partir de la Educación Patrimonial en las clases de Ciencias Sociales. Un estudio de 
caso, centrándonos, aquí, en los bloques del sistema de categorías vinculados con «qué se enseña» de educación para la ciudadanía en las clases de Ciencias Sociales y contrastándolo con lo que dice el alumnado.

En líneas generales, tras hacer una breve presentación del diseño de investigación, y fruto de la sistematización implementada durante el proceso de análisis cualitativo, se muestran los resultados derivados de la estrategia metodológica de «doble triangulación» diseñada para contrastar las observaciones del investigador con las opiniones del docente y de los estudiantes que formaban parte del grupo al que impartía las clases; en este sentido, siguiendo las propuestas de autores como Kelle (2002), asumimos un enfoque que nos permite contrastar la intersubjetividad y asegurar la credibilidad de nuestro proceso de investigación cualitativa. Centramos nuestro análisis de la praxis en la labor de un profesor "posmoderno" -según denominación utilizada por De Castro, 2016-, que se caracteriza por adoptar un enfoque curricular interdisciplinario y multidisciplinario, en el que alumnado y profesorado trabajan juntos para analizar y comprender el mundo desde el presente.

Para ello, concretamente, hemos analizado doce sesiones de la asignatura Ciencias Sociales de un curso de $2^{\circ}$ de ESO, en un centro de secundaria. A lo largo de estas sesiones se desarrolló el bloque temático "Al-Ándalus y los reinos cristianos", establecido en la Orden de 10 agosto de 2007, por la que se desarrolla el currículo correspondiente a la Educación Secundaria Obligatoria en Andalucía. La metodología empleada se caracteriza por ser una metodología activa y cooperativa, como es el llamado "aprendizaje basado en proyectos" (Pozuelos, 2007). Los proyectos realizados en torno a este núcleo temático fueron los siguientes: La Sevilla islámica, La mujer cristiana en la Edad Media, La mujer musulmana en la Edad Media, El escudo de España y los reinos medievales, El camino de Santiago, constituyendo el alumnado cuatro grupos de trabajo, que denominamos G.D.1, G.D.2, G.D.3-4 y G.D.5. Los alumnos/as que desarrollaron los proyectos de La mujer cristiana en la Edad Media y La mujer musulmana en la Edad Media se agruparon en el G.D.3-4 por la proximidad temática de sus proyectos.

\section{DISEÑO Y MÉTODO}

\subsection{Objetivos}

Como se indicó en la introducción, la presente investigación proviene de una investigación doctoral más amplia cuyo objetivo principal es definir un catálogo de buenas prácticas educativas del profesorado innovador de Ciencias Sociales de la ESO, en relación con el patrimonio como contenido pedagógico para la formación de una ciudadanía crítica, responsable y participativa; y analizar en profundidad un caso cercano a un modelo deseable.

Siendo los objetivos específicos:

- Conocer el pensamiento del profesorado innovador acerca de cómo formar ciudadanos/as a través del contenido patrimonial en la materia de Ciencias Sociales.

- Analizar las prácticas de aula del profesorado innovador respecto a la enseñanza del patrimonio y de la ciudadanía y contrastarlas con las concepciones.

- Detectar los obstáculos que tiene el profesorado innovador en la enseñanza del patrimonio y de la ciudadanía en las clases de Ciencias Sociales.

- Como consecuencia de lo anterior, establecer un catálogo de buenas prácticas educativas en educación patrimonial y educación para la ciudadanía en la materia de Ciencias Sociales

\subsection{Participantes}

En primer lugar, hemos de decir que esta investigación se basa en un estudio selectivo, por ello, se realizó un cuestionario inicial de preguntas cortas y valoración múltiple al profesorado de 
Ciencias Sociales, Geografía e Historia de Educación Secundaria Obligatoria que participa en proyectos de innovación educativa, cuyas prácticas de aula se alejan del llamado modelo tradicional. Tras el análisis del cuestionario inicial, realizamos una entrevista exploratoria de concepciones a los seis docentes de perfil de respuesta más deseable según la secuencia planteada en nuestra "hipótesis de progresión", seleccionando con ello el caso para la observación de la praxis docente. Esta hipótesis se ha diseñado basándonos en las gradaciones diseñadas por Cuenca (2003), Matozzi y Ávila (2009) y Ávila (2001), en relación con el patrimonio; y en García Pérez (2002), Schulz (2012) y Schugurensky (2012), en relación con la educación para la ciudadanía, estructurándose dicha progresión en tres niveles siendo el último el nivel que tomamos como referente para el diseño del perfil deseable.

De esta forma, nuestro estudio de caso se encuentra representado por el docente VPG, profesor de Ciencias Sociales, Geografía e Historia de los cursos $1^{\circ}-4^{\circ}$ de Educación Secundaria Obligatoria en el centro IES Castillo de Luna de La Puebla de Cazalla (Sevilla - Andalucía España).

\subsection{Sistema de categorías}

Tanto para orientar el proceso de recogida y organización de la información como para su análisis, se elaboró un sistema de categorías cuya estructura general en categorías, subcategorías, indicadores y descriptores toma como referencia investigaciones anteriores como las de Cuenca (2003, 2010), Lucas (2012), Delgado-Algarra y Estepa-Giménez (2015, 2016), Estepa (2013), y cuya estructura específica bebe de múltiples fuentes. Las categorías del sistema, que derivan de un marco de referencia, son tres: enseñanza del patrimonio, enseñanza de ciudadanía, enseñanza de la educación para la ciudadanía a través del patrimonio. Sin embargo, en esta publicación nos centraremos solamente en los indicadores relacionados con una de las subcategorías de la categoría "enseñanza de ciudadanía", concretamente: «qué enseña el profesor innovador de educación para la ciudadanía» y sus indicadores (tabla 1), para cuyo contenido se tomaron otras fuentes de referencia como Eurydice (2012) y la Evaluation of Educational Achievement [IEA] (Schulz et al., 2008).

TABLA 1. Subcategoría e indicadores vinculados al qué enseña de educación para la ciudadanía el profesor innovador en Ciencias Sociales, Geografía e Historia

\begin{tabular}{|c|c|}
\hline Subcategoría & Indicadores \\
\hline \multirow{5}{*}{$\begin{array}{l}\text { Qué enseña el profesor } \\
\text { innovador de educación para la } \\
\text { ciudadanía en CCSS }\end{array}$} & $\begin{array}{l}\text { Derechos individuales y conocimientos de las instituciones } \\
\text { estatales y civiles }\end{array}$ \\
\hline & Principios cívicos \\
\hline & Identidades cívicas \\
\hline & Interconexiones cívicas \\
\hline & Participación cívica \\
\hline
\end{tabular}

\subsection{Instrumentos y procedimiento de recogida y categorización de la información}

A continuación, se presentan los instrumentos utilizados para la recogida de información:

Observación de la praxis docente: Las clases objeto de investigación fueron grabadas mediante grabadora de audio; asimismo, se tomaron notas puntuales en el diario de la investigadora, ya que esta estaba presente en el aula como miembro no participante. La información registrada mediante grabación y transcrita, al igual que la del resto de instrumentos de la fase cualitativa, se categorizó mediante el programa Atlas.ti.

Grupos de discusión discente: Pese a que la presente investigación se centra fundamentalmente en el docente, conocer el pensamiento del alumnado en relación con el 
desarrollo de las clases nos permite contrastar la interpretación emergente de la primera triangulación (entrevista inicial - observación de aula). Para ello, nos reunimos en grupos de discusión discentes coincidentes con los grupos de trabajo establecidos temáticamente en el desarrollo de las clases observadas. El guion diseñado como guía para recoger la información de esta fase, a pesar de ser un guion abierto, fue ideado en la etapa final de recogida de información, debido a que toma como referente la praxis docente, todo ello tomando siempre como precedente el esquema propuesto en el sistema de categorías. En el anexo 1 recogemos dicho guión, concretamente las preguntas que hacen referencia a la categoría qué enseña el profesor innovador de educación para la ciudadanía en CCSS, que -como se ha dicho- es la que desarrollamos en esta publicación.

Entrevista inicial de concepciones y entrevista final de reflexión docente: la primera, para conocer las concepciones y seleccionar el caso, y la segunda, para conocer la reflexión del docente sobre su propia práctica; sin embargo, puesto que la información obtenida mediante estos instrumentos no se utiliza en el presente artículo no se profundizará más en ello.

Pasando a los criterios de categorización de las unidades de información obtenidas a partir de los dos primeros instrumentos: en cuando a la observación, se indica el número de sesión (O.3), seguido del número de anotación registrada en el programa Atlas.ti (Quotation: 3:2). Ejemplo: "información" (O5 Quotation: 5:2). Sin embargo, en relación con los grupos de discusión, se indica el número del grupo (G.D.1), seguido del número de pregunta del guión (P.1) y el número de anotación registrada en el programa Atlas.ti (Quotation 1:36). Ejemplo: "información" (G.D.1/P.1/Quotation 1:36).

\subsection{Procedimiento de análisis de información}

Tomando como modelo de análisis el modelo de «doble triangulación» utilizado por DelgadoAlgarra y Estepa-Giménez $(2015,2016)$ (Figura I), la investigación que se presenta tiene un enfoque multimetódico que nos permite contrastar la intersubjetividad y asegurar la credibilidad de nuestro proceso de investigación cualitativa.

GRÁFICO 1. Esquema de doble triangulación utilizado en el estudio.

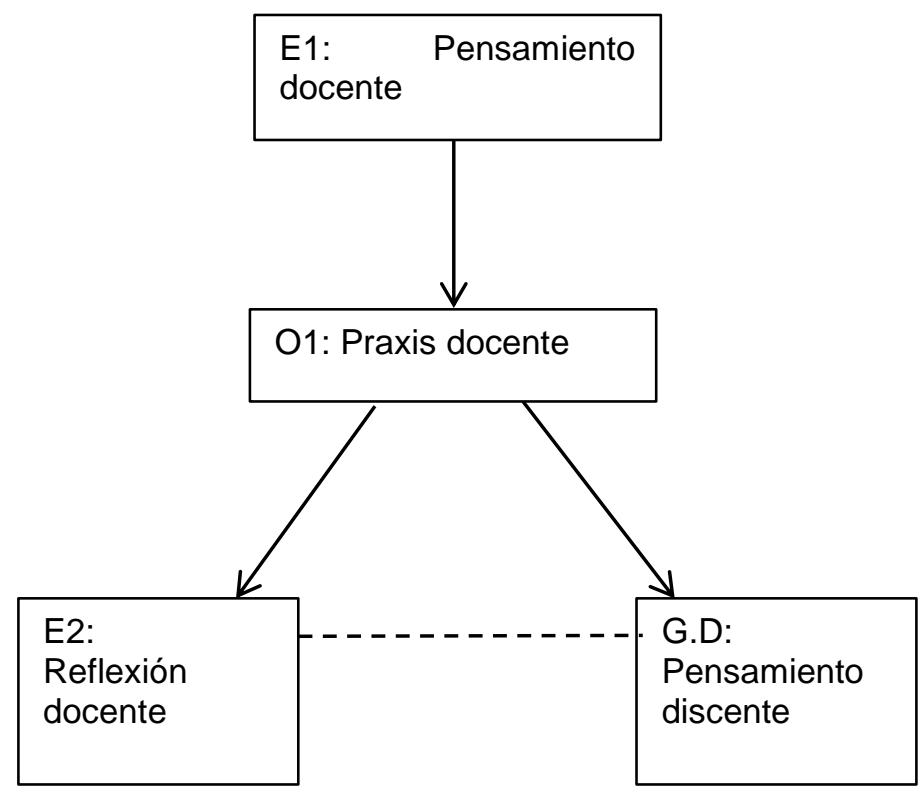

Como se muestra en el Gráfico 1, mientras en la primera triangulación (E1-O1) se lleva a cabo un proceso de interpretación que nos permite destacar las convergencias y divergencias 
existentes entre las concepciones del docente y su práctica bajo el paraguas del sistema de categorías, en la segunda triangulación (G.D-O1-E2), reforzamos la validez y la fiabilidad de nuestras interpretaciones con el apoyo de la percepción que los estudiantes tienen del desarrollo de las clases y la interpretación que el docente hace de dichas percepciones.

\section{RESULTADOS}

A continuación, procederemos a hacer una síntesis del análisis de cada indicador, con el fin de profundizar en los aspectos recogidos producto de la observación y de los grupos de discusión discentes.

En cuanto al indicador "derechos individuales y conocimientos de las instituciones estatales y civiles", ni en la observación de la práctica docente ni en los discursos discentes registrados en los grupos de discusión, se recogen evidencias; sin embargo, observamos que se alude al indicador "principios cívicos" con frecuencia a lo largo de las sesiones de aula, destacando los descriptores igualdad, libertad, cohesión social, dignidad y justicia social.

Así, temas como la violencia machista y la desigualdad de género son trabajados en varias sesiones. En ellas, veíamos cómo el docente daba importancia a que su alumnado fuera consciente de que el problema machista ha sido una constante que se ha perpetuado a lo largo del tiempo, por lo que guiaba a los grupos que trabajaban sobre la mujer cristiana y sobre la mujer musulmana, respectivamente a que investigaran la situación social de la mujer en la Edad Media en ambas culturas.

"Se debe subrayar como una constante histórica. Va[n] pasando los tiempos. Y una constante transcultural. Transcultural quiere decir que no sólo en el tiempo sino también en el espacio, donde viven diferentes culturas ha sido una constante... una continuidad, que los hombres se han apañado de tal forma y manera que siempre ha[n] dominado a la mujer... En muy pocas excepciones, en muy pocas excepciones históricas, la mujer ha tenido cotas de igualdad que... por ejemplo, en la actualidad ciertos países. Hay ciertos países, países occidentales, más desarrollados, con democracias más avanzadas... hombres y mujeres son iguales. Pero no es verdad que [en] nuestra democracia actual exista esta igualdad ”(O.12/Quotation 11:1).

Este posicionamiento se refuerza al analizar los grupos de discusión discentes. El G.D.2 muestra su interés en este tema como una problemática social actual (G.D.2/Quotation 2:23) y el G.D.3-4 reflexiona sobre cuál es la situación social de la mujer actual y qué roles se perpetúan, relacionando su trabajo con el problema de la conciliación laboral:

"Investigadora: ¿Y creéis que, en cierta manera, en la actualidad, todavía quedan cosas de antes? ¿Hay un paralelismo entre antes y ahora?

Alumna: Sí.

Alumna: Yo creo que sí que, aunque se ha evolucionado... no tanto...

Alumna: En realidad tampoco ha cambiado tanto... En los trabajos... muchas de las mujeres se siguen dedicando a cuidar a los niños, haciendo de comer y a limpiando la casa.

Alumna: Pero no tiene por qué. Puede trabajar.

Alumna: Hay mujeres que trabajan y también hacen el trabajo de casa. Igual que antes, trabajaban en el campo y en casa" (G.D.3-4 /Quotation 3:43).

Así mismo, el profesor insiste en analizar las causas del machismo y destaca la necesidad de educar en igualdad para poder erradicar este problema:

"Creo que es importante que, en la escuela, sin que tenga que venir nadie a dar una charla... Y sin que tenga que ser una clase de ética o de ciudadanía, sino que sea un tema transversal. Quiero decir que todas las materias sociales se prestan a tratarlo... Me ha gustado mucho la historia... que recordéis que ya en el siglo XIV hubo mujeres que se acordaban de que había 
otras mujeres que, por el hecho de ser mujer, es decir por ser diferente...Evidentemente el hombre y la mujer son diferentes. Pero la diferencia corporal, física, no puede ser motivo de desigualdad. La diferencia no se puede convertir en la causa de que unos sean superiores de[sic] otros, ¿no?... Hay que educar en la igualdad, respetando las diferencias” (O.11/Quotation 10:4).

Profundizando en las respuestas de los grupos de discusión discente, extraemos una serie de anotaciones que son coherentes con las preocupaciones explicitadas en la observación de aula. De tal forma que una alumna de G.D.3-4 reconoce que el estudio de este tema le ha llevado a replantearse comportamientos y actitudes con el fin de cambiarlos, asumiendo su compromiso hacia una sociedad más igualitaria: “... a ser buen ciudadano... no tanto para fuera, pero... es que no sé cómo decirlo... para dentro, a comportarme y a pensar, yo, en mi vida" (G.D.3-4 /Quotation 3:47).

Por otro lado, la cohesión social tiene una fuerte presencia en el discurso de los estudiantes. Todos ellos destacan el respeto a las normas para poder convivir, pero insisten en que estas normas deben tener en cuenta las diferentes realidades culturales que conviven en nuestro país. Conforme a esto, cuando se les pregunta por el "problema de Cataluña" en relación a la independencia, entienden que se pueda demandar esta independencia si desde el estado central no se valora ni se respeta los rasgos identificativos de esta comunidad: "Yo no lo veo como un problema. Yo creo que si se quieren independizar es porque no tienen importancia en España... Porque por otra cosa no puede ser" (G.D.2/ Quotation 2:30).

Nuevamente, hay una total coherencia entre el discurso discente y la práctica docente, ya que esta idea pudimos registrarla en varias sesiones de la O1. El docente destacaba que una sociedad igualitaria y justa tiene un alto grado de cohesión social, ya que los integrantes que forman parte de un mismo colectivo tienen intereses comunes a pesar de que existan diferencias entre ellos. (O6/Quotation: 6:15).

Por otro lado, es importante señalar el planteamiento discente de valorar y "cuidar lo nuestro", refiriéndose a poner en valor su patrimonio local; lo cual refleja la importancia de dignificar su identidad local como componente fundamental para ser un ciudadano democrático y comprometido con su entorno:

"Investigadora: Si lleváis algún proyecto para proteger algún elemento patrimonial, como por ejemplo el castillo de Luna. Si en clase hacéis algún proyecto para protegerlo o rehabilitarlo, ¿os enseñaría eso ciudadanía?

Alumna: Yo creo que sí.

Alumna: Estamos respetando, estamos haciendo... Cuidando lo nuestro.

Alumna: Cuidando lo nuestro estamos respetando... ¡ay, que no me sé explicar!” (G.3 Quotation 3:26).

Por último y sin encontrar vinculación con la praxis, destaca la importancia que los estudiantes dan a la libertad individual para ser un buen/a ciudadano/a, señalan que la libertad individual está por encima de lo marcado por la ley: Tú tienes la libertad de hacer lo que quieras (G.3 Quotation 3:24).

En relación al indicador "identidades cívicas", en la praxis docente, no se encontró ninguna evidencia que pueda adscribirse al primer indicador "autoimagen cívica"; por el contrario, el segundo indicador, "interconexiones cívicas", era el que mayor número de anotaciones había recibido dentro de esta segunda subcategoría de análisis, destacando el papel de la tolerancia a la diversidad y centrándose, principalmente, en dos problemáticas actuales a las que pueden dar respuesta a través de la investigación de sus proyectos. Una es la independencia de Cataluña y la otra el radicalismo islámico y la visión que los medios de comunicación dan de él. En relación a la primera, el docente, en la sesión seis, hablaba del sentimiento de identidad de una parte de la comunidad catalana, que no sentía que esa identidad estuviera reflejada en el escudo actual a pesar de que Cataluña sí que está representada: "Para algunos catalanes ese escudo no les representa. A 
pesar de que alguno puede decir, pero sí que estáis representados. Pero bueno, ellos tienen un sentimiento y ese escudo no les representa" (O6/ Quotation: 6:14). Vemos aquí, claramente, cómo el docente tenía un marcado interés en que su alumnado adquiriese un sentimiento identitario en relación a sus diferentes comunidades cívicas. Sentimiento que, en los grupos de discusión discente, pudimos comprobar que era asimilado cuando preguntamos acerca de si el estudio de su tema les ayuda a ser ciudadanos, a lo que responden afirmativamente.

"Investigadora: Analizando imágenes, documentos... Por ejemplo, vosotros habéis analizado el escudo de España, eso es una imagen, ¿no? ¿Os ha ayudado a aprender a ser ciudadano?

Alumno: A respetar los distintos lugares de nuestro país" (G.2 Quotation 2:29).

Con respecto al problema del fundamentalismo islámico, veíamos cómo el profesor pretendía superar la visión que los medios de comunicación dan de la sociedad islámica, centrada en los tópicos, intentando que los alumnos conozcan las diferentes realidades que forman esta cultura, y que pudieran comprobar por ellos mismos que, en muchos casos, no está tan alejada de nuestros valores y sentimientos occidentales:

\begin{abstract}
"Nunca en los medios de comunicación, los musulmanes salen bien parados. Por el contrario, la imagen que se da de los musulmanes es de gente mala. Pero os advierto una cosa, el país del mundo con mayor número de musulmanes se llama Indonesia. Indonesia es un país [que] está debajo de China, de Vietnam, ¿vale? Y, en consecuencia, los indonesios, si les viésemos su cuerpo físico diríamos que son chinos... ¿Quién iba pensar que el país con mayor número de musulmanes del mundo, su gente iba a tener aspecto de oriental, con los ojos achinados y de esa forma? ¿Verdad que no? ¿A que cuesta trabajo imaginárselo? Pues así es. (Quotation: 11:2 PD: Transcripción O12)
\end{abstract}

En relación con el alumnado vemos una vez más la trasposición de estas ideas a sus aprendizajes, de forma que cuando preguntamos si consideran que las clases de Ciencias Sociales les ayudan a formarse como ciudadanos, dicen que sí, puesto que adquieren conocimientos de otras culturas para poder entenderlas mejor y conectar con ellas.

"Investigadora: Vale. Bien, entonces, ¿creéis que las clases de Ciencias Sociales os ayudan a ser ciudadanas?

Alumnas: Sí.

Investigadora: ¿Por qué?

Alumna: Porque si a lo mejor entiendo más la cultura de otra persona, a lo mejor yo puedo ser más tolerante con ella.

Alumna: Es que, por ejemplo, ahora estamos dando la Edad Media, los musulmanes, y nosotros no sabemos mucho de los musulmanes y a partir de ahí podemos entenderla mejor y al estar conviviendo con ella podemos entenderla y convivir mejor con ella" (G.5 Quotation 4:21)

En el caso del alumnado el indicador "autoimagen cívica", como decíamos, sí que tiene presencia en sus planteamientos, refiriéndose a las experiencias individuales que el individuo mantiene en su comunidad. Creen que conocer el entorno en el que viven es fundamental para ser buenos ciudadanos, favoreciendo su socialización.

"Investigadora: ¿Creéis que las clases de Ciencias Sociales os ayudan a ser ciudadanos? Alumnos/as (todos/as): Sí.

Alumna: Mucho, porque nosotros tenemos otra idea de dónde es donde nosotros vivimos... de la ciudadanía... Y no. A nosotros nos aporta mucho porque nos da otra idea de lo que es, más o menos, en verdad la civilización nuestra" (G.1 Quotation 1:51) 
Por último, el indicador "participación cívica" es el que mayor peso tiene tanto en la praxis como en el pensamiento discente, coincidiendo en ambos casos que el debate y la participación del alumnado en el aula es una constante en el desarrollo de las clases, siendo ampliamente puesta en práctica para adquirir conocimientos cívicos. Los discentes dejan claro que en la praxis docente la toma de decisiones y la participación del alumnado es una constante, habiendo una coherencia plena con lo analizado en la O1 (O13/Quotation: 12:4)

"Alumno: Y además nosotros... la clase que nos dan, no viene un profesor a soltarnos el rollo de los apuntes. Nosotros utilizamos la democracia para decidir las cosas" (G.1 Quotation 1:24)

Además, para el alumnado lo más interesante para poder adquirir conocimientos y comportamientos ciudadanos es la participación en el aula y poder expresar sus opiniones en relación a distintos temas.

"Investigadora: ¿Y de lo que hacéis y tratáis en clase qué os enseña a ser ciudadano?

Alumno: Por ejemplo, lo que estamos haciendo de exponer porque aprendes a no tener miedo escénico y a explicarte en general.

Alumna: Yo, más o menos, digo lo mismo que Juan porque estamos expresando nuestra opinión. Y, además, estamos manifestándonos para un tema" (G.1 Quotation 1:37).

Pese a que el docente tuvo que intervenir para poner orden: "creo que debéis de aprender a guardar el turno" (O13/Quotation: 12:4), los discentes son conscientes de estos conflictos y aseguran que aprenden a gestionarlos, desarrollando actitudes de participación democrática.

"Investigadora: ¿Y qué aspectos negativos veis en trabajar de esta manera?

Alumno: Que surgen conflictos dentro del grupo.

Investigadora: ¿Y llegáis a solucionar estos conflictos?

Alumno: Sí” (G.2 Quotation 2:26).

Pero, además, comprobábamos en la $\mathrm{O} 1$ que la participación se trabajaba también desde el punto de vista de los contenidos. El docente proponía y aceptaba aquellas aportaciones que implica la participación ciudadana para trasformar la sociedad, como es el movimiento feminista. Así, uno de los grupos se había propuesto buscar a la primera mujer feminista. Aportación que el docente acogía de buen grado y animaba a que continuaran en esa línea de investigación: "Entonces estáis hablando de Cristina de Pisan, ¿no? La primera mujer feminista de la historia...Muy bien”" (O11/Quotation: 10:1). En otra ocasión el docente destaca el papel activo y reivindicativo de algunas mujeres hasta el punto de hacerse pasar por hombres para avanzar socialmente:

"Esto es muy interesante porque a lo largo de la historia han sido muchas, muchísimas las mujeres que, para avanzar, prosperar económicamente, avanzar socialmente se han hecho pasar por hombres. Pero muchísimas mujeres que, para intentar ser libres, entre comillas, han tenido que hacerse pasar por hombre. Vistiéndose como hombre, pelándose, adoptando las formas propias de hombre... Esto tiene... No sé qué os parece, pero es increíble, ¿no?" (O11/Quotation: 10:9).

En relación con lo declarado por los discentes, comprobamos que estos contenidos son asimilados y, a través de ellos, adquieren comportamientos y actitudes propias de una ciudadanía participativa y democrática, comprometiéndose con su realidad y desarrollando un pensamiento reflexivo que cuestiona las injusticias y toma medidas para llevar a cabo cambios sistémicos.

"Investigadora: ¿Qué contenidos de todos los que habéis trabajado os han resultado más interesantes?

Alumna: El machismo y que había feministas desde antes, que no es algo nuevo. 
Alumna: Que ya se luchaba contra el machismo desde hace tiempo.

Alumna: Que antes las mujeres ya eran maltratadas... que viene de antes.

Investigadora: ¿Y qué es el feminismo para vosotras?

Alumna: El luchar por la igualdad de género.

Investigadora: ¿Todas las mujeres actuales somos feministas o no tiene por qué?

Alumna: Hay algunas que no.

Alumna: No tiene por qué.

Alumna: Aunque su marido la esté...

Alumna: Pegando...

Alumna: No.

Alumna: Maltratando...

Alumna: No... no quiero decir eso... Tratándole como inferior, ella se cree que está así bien y que tiene que ser así.

Investigadora: Pero el feminismo es un movimiento que va más allá, ¿no? Luchar contra el maltrato no es sólo feminismo. El maltrato es un delito. El feminismo es luchar por la igualdad con acciones que lleven a ello.

Alumna: Sí, porque tú puedes decir que eres feminista y después no hacer nada" (G.3 Quotation $3: 40)$.

\section{DISCUSIÓN Y CONCLUSIONES}

Tras la exposición de los resultados obtenidos, constatamos que en esta investigación se observan diferencias con respecto a investigaciones precedentes. Según Cotton (2001), los contenidos que se proponen en las aulas en relación con la educación para la ciudadanía se presentan desconectados de los problemas reales a los que se enfrentan los estudiantes, lo que desencadena su pérdida de interés e implicación. Gómez Rodríguez (2007) añade que, a pesar de que la mayoría del profesorado afirma que la enseñanza de la ciudadanía debe incluir el tratamiento de temas que son socialmente controvertidos y polémicos - tal y como hemos visto que hace el docente investigado-, en realidad, estos temas se evitan por considerar que tienen connotaciones políticas partidistas, o porque podrían resultar inaceptables y mal vistos para algunos sectores de la población. Por lo que Navarro (2012) y De Alba (2007), sostienen que la educación para la ciudadanía que desarrolla la mayor parte del profesorado de Ciencias Sociales es una ciudadanía pasiva y reproductora del sistema.

Sin embargo, como acabamos de comprobar, ante la categoría de qué enseña el profesor innovador de educación para la ciudadanía en CCSS, nos encontramos ante un docente comprometido con las problemáticas sociales actuales, que busca que el alumnado pueda desarrollar un pensamiento crítico que le lleve a comprender su realidad para comprometerse y participar en su comunidad (Pineda-Alfonso, 2015).

En este sentido, en el pensamiento, praxis y reflexión discente se refleja la importancia de adquirir y respetar los principios cívicos que son garantía de cualquier democracia (libertad, igualdad, dignidad, cohesión social y justicia social) y desarrollar conexiones cívicas con diferentes comunidades. Así, el alumnado aprende a tomar decisiones y son conscientes de la importancia de participar en acciones sociales y comunitarias, que lleven a cambios sistémicos para lograr una sociedad más equitativa y justa. Sin embargo, pese a la presencia de las "interconexiones cívicas", durante la observación de aula, no se recogen informaciones explícitas vinculadas con la "autoimagen cívica", pero sí implícitas, ya que a través del trabajo de exploración y de indagación en los temas trabajados, reflexionan acerca de su identidad individual dentro de su realidad social. Así lo vimos con los grupos de trabajo sobre los temas de la mujer cristiana y musulmana, que llegaron a plantearse y cuestionar comportamientos propios, y que están normalizados socialmente como discriminatorios y generadores de desigualdad.

Todo lo anterior nos lleva a concluir que el profesor que hemos caracterizado en líneas anteriores (y que calificamos anteriormente como "posmoderno"), a través de los proyectos que se 
desarrollan en torno a los referentes patrimoniales, intenta que su alumnado entienda y pueda dar respuesta a problemas actuales relacionados con la organización económica, política, social y cultural del presente, pero siempre comparando presente-pasado, con la intención de que sus estudiantes puedan desarrollar una actitud crítica y reflexiva. Es por ello que, para este docente, la participación es el contenido fundamental de educación para la ciudadanía que se debe desarrollar en el aula, aplicando estrategias metodológicas centradas en la investigación escolar, favoreciendo así un entorno adecuado para que sus estudiantes puedan mejorar su aprendizaje en su dimensión académica, humana y social (Hernández de la Torre y Medina, 2014).

Así, nos mostramos de acuerdo con De Castro (2016), cuando afirma que el profesor posmoderno debe entender la educación desde su capacidad transformadora y cuestionando en todo momento "la educación exclusivamente reproductiva, cognitivista, orientada solo al conocimiento intelectual-racional” (Barragán Rodríguez, 2005, p.56) se supera así el simple conocimiento del patrimonio y se sitúa al alumnado en su realidad presente para saber actuar e influir en ella. De ello se deduce que este modelo de profesor tiene muy presente que el fin último de todo sistema educativo es la formación para una ciudadanía democrática, y, como tal, la participación y el compromiso social son los dos comportamientos básicos que debe aprender su alumnado.

\section{Referencias}

Ávila, R. M. (2001). Historia del arte, enseñanza y profesores. Sevilla: Díada.

Barragán Rodríguez, J. M. (2005). Educación artística, perspectivas críticas y práctica educativa. En Marín Viadel, R. (Ed.). Investigación en educación artística: temas, métodos y técnicas de indagación sobre el aprendizaje y la enseñanza de las artes y culturas visuales (pp. 43-80). Granada: Universidad de Granada.

Cuenca López, J. M. (2003). Análisis de concepciones sobre la enseñanza del patrimonio en la educación obligatoria. Enseñanza de las Ciencias Sociales: revista de investigación, 2, 37-45. Recuperado de: https://goo.gl/Gruv2i

Cuenca López, J. M. (2010) El Patrimonio en la didáctica de las Ciencias Sociales: análisis de concepciones, dificultades y obstáculos para su integración en la enseñanza obligatoria. Universidad de Huelva.

De Alba, N. (2009). Parlamento Joven: una experiencia de educación para la ciudadanía democrática. Investigación en la Escuela, 68, 73-84. Recuperado de https://goo.gl/d1FGLi

De Castro, P. (2016). Cartografía autoetnográfica de una genealogía de programas de educación patrimonial desde la perspectiva del aprendizaje basado en proyectos y la investigaciónacción (Tesis doctoral). Universidad de Valladolid, Valladolid.

Delgado-Algarra, E. (2014). Educación para la ciudadanía en la enseñanza de CCSS y su vinculación con las dimensiones de la memoria. Estudio de caso (Tesis doctoral). Universidad de Huelva, Huelva.

Delgado-Algarra, E. J. y Estepa-Giménez, J. (2015). Educación ciudadana y memoria histórica en la enseñanza de las Ciencias Sociales. Enseñanza de las Ciencias Sociales, 14, 97-109. Recuperado de https://goo.gl/LnaE4A

Delgado-Algarra, E. y Estepa-Giménez, J. (2016). Ciudadanía y memoria histórica en la enseñanza de la historia: análisis de la metodología didáctica en un estudio de caso en ESO. Revista de Investigación Educativa, 34(2), 521-534. DOI: 10.6018/rie.34.2.224891. Recuperado de https://goo.gl/pZiYpf

Delgado-Algarra, E. y Estepa-Giménez, J. (2017). Educación ciudadana y dimensiones de la Memoria en la enseñanza de Ciencias Sociales: investigación sobre las concepciones del profesorado de Educación Secundaria de Huelva y provincia. Educación XX1, 20(2), 259-278. DOI: $10.5944 /$ educXX1.11926. Recuperado de https://goo.gl/TtKpb7 
Delors, J. (1996). La educación o la utopía necesaria. En J. Delors (Presidente), Informe a la UNESCO de la Comisión Internacional sobre la educación para el siglo XXI. La Educación encierra un tesoro. Madrid: Santillana y Ediciones Unesco.

Estepa-Giménez, J. (2013) La educación patrimonial en la escuela y el museo: investigación y experiencias. Huelva: Servicio de Publicaciones de la Universidad de Huelva.

García Pérez, F. F. (2002). Concepciones de los alumnos y conocimiento escolar. Un estudio en el ámbito del medio urbano. Enseñanza de las Ciencias Sociales, 1, 17-25. Recuperado de https://goo.gl/RE4j1e

Hernández De La Torre, E. y Medina, P. (2014). Análisis de los obstáculos y barreras para el cambio y la innovación en colaboración en los centros de secundaria: estudio de caso. Revista de Investigación Educativa, 32(2), 269-287. DOI: 10.6018/rie.32.2.172041. Recuperado de https://goo.gl/Q274Ai

Kelle, U. (2002). Sociological Explanations between Micro and Macro and the Integration of Qualitative and Quantitative Methods. Forum Qualitative Sozial for schung / Forum: Qualitative Social Research, 1(2), 43. DOI: 10.17169/fqs-2.1.966.

Lucas, L. (2013). Las concepciones del profesorado de Educación Secundaria Obligatoria de Ciencias Sociales sobre el patrimonio como instrumento para formar ciudadanos y las concepciones que maneja sobre ello el alumnado al que da clase (Trabajo Fin de Máster). Universidad de Huelva, Huelva.

Lucas, L. y Estepa, J. (2016a) Identidad, valores cívicos y participación ciudadana en la Didáctica del Patrimonio. Aprendizaje y enseñanza. En S. Molina Puche, A. Escribano-Miralles y J. Díaz-Serrano (Ed.), Patrimonio, identidad y ciudadanía en la enseñanza de las Ciencias Sociales (pp. 89-100). Murcia: Servicio Publicaciones universidad de Murcia.

Lucas, L. y Estepa, J. (2016b) El patrimonio como instrumento para la formación de la ciudadanía crítica y participativa. Investigación en la Escuela, 89, 35-46. Recuperado de https://goo.gl/Nxfp9t

Matozzi, I. y Ávila, R. M. (2009). La didáctica del patrimonio y la educación para la ciudadanía. En R. Avila, B. Borghi y M. Matozzi (Ed.), Léducaziones alla cittadinanza europea e la formazione degli insegnanti. Un progetto educativo per la "strategia di Lisbona" (pp. 327352). Bologna: Pàtron Editore.

Tutiaux-Guillon, N. (2003). L'Histoire enseignée entre coutume disciplinaire et la formation de la conscience historique. En N. Tutiaux-Guillon y D. Nourrison (Ed.), Identités, mémoires, consciente historique (pp. 27-39). Francia: Université de Saint Étienne.

Pozuelos, F. (2007). Trabajo por proyectos en el aula: descripción, investigación y experiencias. Sevilla: Cooperación Educativa.

Pineda-Alfonso, J. A. (2105). Educar para la ciudadanía trabajando con temas controvertidos en Educación Secundaria Obligatoria. Revista de Investigación Educativa, 33(2), 353-367. DOI: 10.6018/rie.33.2.208441. Recuperado de https://goo.gl/TekUHy

Schugurensky, D. (2012). El profesorado y la enseñanza de la educación ciudadana. En N. De Alba, F. F. García y A. Santisteban (Eds.), Educar para la participación ciudadana en la enseñanza de las Ciencias Sociales. XXIII Simposio Internacional sobre la Didáctica de las Ciencias Sociales. Sevilla: Diada Editora.

Schulz, W. (2012). Educación para la ciudadanía y participación ciudadana. Una presentación del estudio ICCS 2009 y sus resultados. En N. De Alba, F. F. García y A. Santisteban (Eds.), Educar para la participación ciudadana en la enseñanza de las Ciencias Sociales (I) (pp. 4763). Sevilla: Diada Editora. 


\section{ANEXO 1: GUION ORIENTATIVO DEL GRUPO DE DISCUSIÓN}

\section{GRUPO DE DISCUSIÓN_3-4: LA MUJER CRISTIANA EN LA EDAD MEDIA Y LA MUJER MUSULMANA EN LA EDAD MEDIA}

PR.3/G.D.3-4: ¿Crees que las clases de Ciencias Sociales te enseñan a ser ciudadano? qué?

PR.4/G.D.3-4: ¿Cuál de las siguientes maneras te sería más útil para aprender qué es ser ciudadano y por

Excursiones y visitas fuera del aula (museos, monumentos, yacimientos arqueológicos.

Analizando imágenes, documentos sobre nuestra constitución, nuestros derechos electorales.

Con el visionado de alguna película o documental sobre movimientos ciudadanos.

Llevando a cabo algún proyecto para proteger un elemento patrimonial en peligro de tu ciudad.

Una charla de un político.

La explicación del profesor.

Simulando un proceso electoral en el aula.

Una charla de un director de museo o de un centro de interpretación de patrimonio.

PR.5/G.D.3-4: ¿Crees que el patrimonio es importante para la sociedad actual? ¿Por qué?

PR.6/G.D.3-4: ¿Crees que investigar y estudiar el patrimonio te ayuda a ser ciudadano? ¿Cómo?

PR.7/G.D.3-4: ¿Cuál piensas que es el comportamiento de un buen ciudadano?

PR.8/G.D.3-4: ¿Cuál piensas que es el comportamiento de un mal ciudadano?

PR.10/G.D.3-4: Imagina que se incorpora una nueva alumna que es musulmana y su familia la obliga a ponerse velo cuando salga a la calle. Después de haber investigado y conocido el significado de llevar velo, ¿qué te parece? ¿harías algo?

PR.11/G.D.3-4: En vuestra presentación decís que las mujeres en la Edad Media tenían que cubrirse la mayor parte del cuerpo, ¿habéis podido investigar el por qué?

De manera similar, el traje de novia actual también cubre bastante el cuerpo de la mujer y también se usa el velo, ¿qué opináis al respecto? ¿este velo también puede significar sumisión respecto al marido?

PR.12/G.D.3-4: ¿Qué contenidos os han parecido más interesantes? ¿Por qué?

PR.13/G.D.3-4: ¿Qué contenidos os han parecido menos interesantes? ¿Por qué?

PR.14/G.D.3-4: ¿Qué contenidos consideras que te ayudan a formarte como ciudadano? ¿Por qué? ¿Qué te ha aportado el estudio de este tema?

PR.15/G.D.3-4: ¿Crees que es mejor olvidar los conflictos del pasado, como, por ejemplo, las guerras o las invasiones de las que nos habla la historia o, por el contrario, crees que es mejor recordarlas y analizarlas con nuestra mirada de ahora? ¿Por qué?

PR.16/G.D.3-4: Imagina que vuestro porfesor os propone ir de excursión y que una compañera dice que no puede ir porque su novio no la deja. ¿Qué os parece? ¿Cómo actuaríais?

PR.17/G.D.3-4: ¿Creéis que de alguna manera el estudio de este tema está relacionado o se puede relacionar con la violencia machista actual? ¿Por qué?

PR.18/G.D.3-4: ¿Creéis que los ciudadanos podemos hacer algo para erradicar la desigualdad de género y la violencia machista? ¿El qué?

¿Y el gobierno? 
PR.19/G.D.3-4: ¿Qué idea se corresponde más con el desarrollo de las clases de Ciencias Sociales? Mi profesor no permite los debates y nunca da su opinión.

Mi profesor no permite los debates y nos da su opinión.

Mi profesor nos permite debatir sin dar su opinión.

Mi profesor nos permite debatir y da su opinión.

PR.20/G.D.3-4: Durante el desarrollo de las clases, ¿te sientes libre para opinar? ¿Por qué?

PR.21/G.D.3-4: ¿Piensas que tu profesor quiere una única opinión o plantea la posibilidad de que puedan existir diferentes opiniones en los debates?

PR.22/G.D.3-4: ¿Tu profesor compara el presente con el pasado? Si la respuesta es "sí" ¿Qué beneficios piensa que esa comparación aporta a tu formación como ciudadano?

PR.23/G.D.3-4: De los recursos que habéis usado en el aula ¿Cuál te ha parecido más interesante y cuál te ha parecido menos interesante?

Libro de texto

Artículos / Textos

Debates

Vídeos

Audios

Otros, ¿cuáles? ¿Por qué?

PR.24/G.D.3-4: De todos los temas que se están trabajando, incluido el tuyo, ¿cuál o cuáles te parecen más interesantes? ¿Por qué? ¿Y menos? ¿Por qué?

PR.25/G.D.3-4: ¿Te gusta esta forma de trabajar investigando? ¿Crees que aprendes más o menos? ¿Qué aspectos positivos tiene? ¿Y negativos?

\section{CÓMO CITAR ESTE ARTÍCULO}

Lucas Palacios, L. y Delgado Algarra, E. J. (2018). Educación para una ciudadanía comprometida en la enseñanza de las Ciencias Sociales: ¿Qué piensa el alumnado de un profesor innovador sobre su aprendizaje? Didáctica de las ciencias experimentales y sociales, 35, 3-16. DOI: 10.7203/DCES.35.12572. 